ISSN 1409-2441

www.ucr.ac.cr
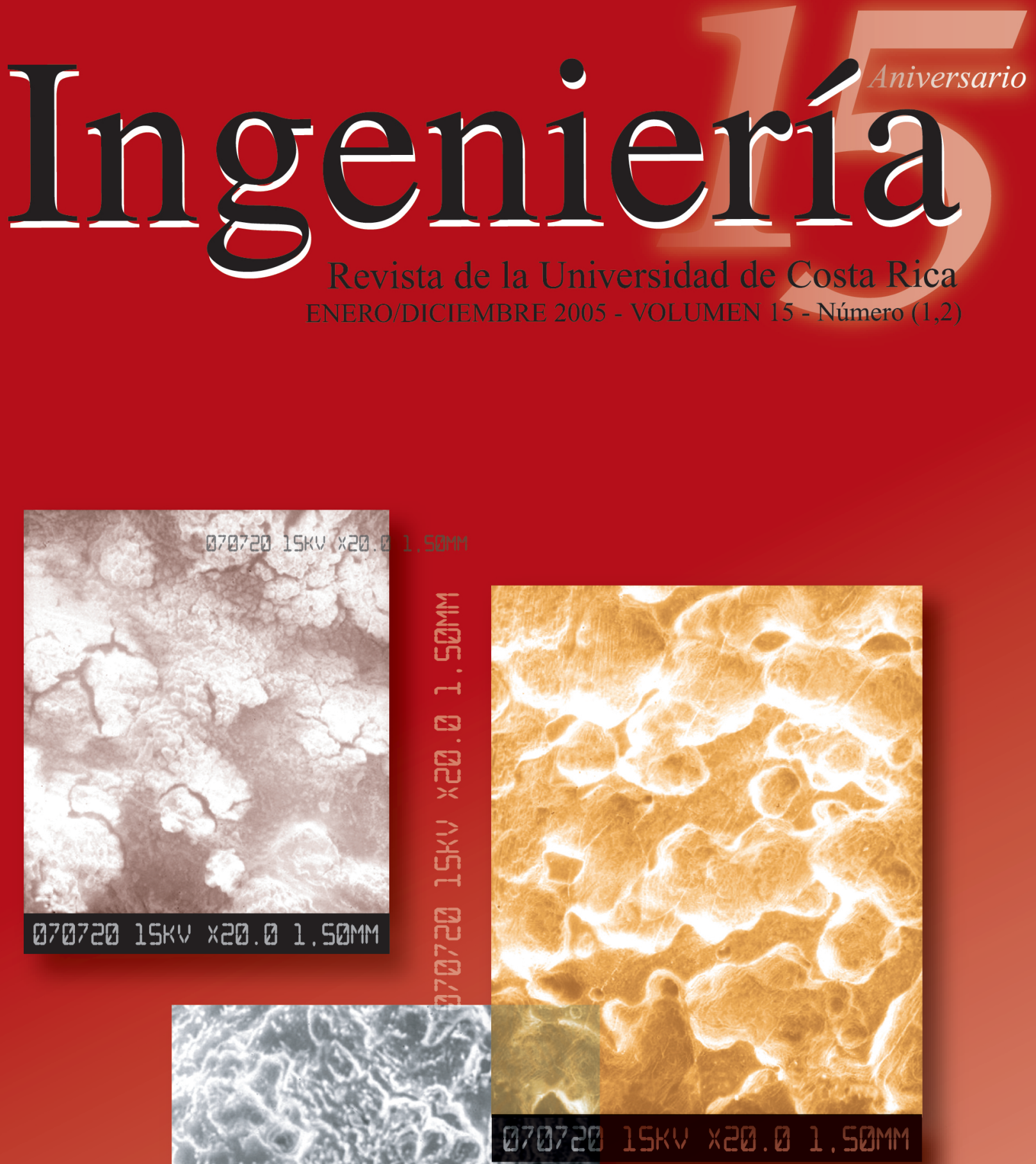

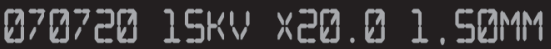

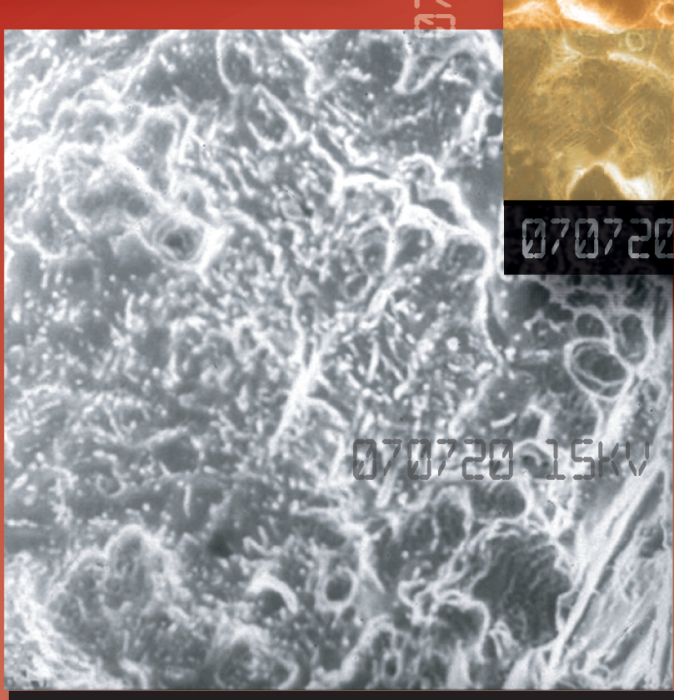

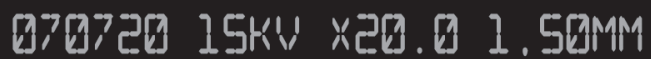




\title{
ACTUALIZACIÓN DEL MÉTODO DE SINTONIZACIÓN DE CONTROLADORES DE ZIEGLER Y NICHOLS
}

\author{
Víctor M. Alfaro Ruiz.
}

\begin{abstract}
Resumen
Se presentan las reglas de sintonización de controladores de Ziegler y Nichols en su forma original y se describen sus características principales. Además, se actualizan estas ecuaciones de sintonización, para su utilización con los controladores PID actuales y los modelos identificados a partir de la curva de reacción del proceso. Mediante un ejemplo se muestra la utilización de las reglas actualizadas.
\end{abstract}

Palabras clave: controladores PID, métodos de sintonización, Ziegler y Nichols

\section{Abstract}

The original Ziegler y Nichols tuning rules and its main characteristics are presented. These tuning rules are updated for use with modern PID controllers and process models obtained from the process reaction curve, an example is presented showing the use of the updated rules.

Key words: PID controllers, tuning methods, Ziegler and Nichols

Recibido: 8 de noviembre del 2004 Aprobado: 2 de junio del 2005

\section{INTRODUCCIÓN}

$\mathrm{Al}$ momento en que Taylor Instrument introdujo al mercado, en 1940, el primer controlador PID, el Fulscope modelo 100, no existía un procedimiento sistemático para la sintonización de los controladores industriales. Hasta esa fecha, los controladores $P, P I$ o $P D$, se sintonizaban, básicamente, mediante procedimientos de prueba y error.

John Ziegler y Nathaniel Nichols, ingenieros de Taylor, se abocaron entonces a la tarea de realizar pruebas experimentales con varios procesos para determinar una forma simple de sintonizar el nuevo controlador. El resultado de ese trabajo, fue el método de sintonización ampliamente conocido como método de Ziegler y Nichols (Ziegler \& Nichols, 1942), el cual despertó reacciones encontradas al momento de su presentación, en la reunión anual de la Sociedad Americana de Ingenieros Mecánicos (ASME) en 1941 (Bickley, 1990).

A pesar de que han transcurrido ya más de sesenta años desde su desarrollo, el método de Ziegler y Nichols se sigue empleando, ya sea en su versión original o con modificaciones. Sin embargo, en la literatura se encuentran con frecuencia errores en su aplicación, o controversias sobre el tipo de controlador PID para el cual fue desarrollado.

Se presenta aquí una actualización de las reglas de sintonización de controladores de Ziegler y Nichols, sin entrar a compararlas con otras técnicas; el artículo está organizado de la siguiente forma: en el apartado 2 se presentan las ecuaciones tal como fueron derivadas originalmente por 
Ziegler y Nichols; en el apartado 3 se analizan las características que definen la aplicación del método; en el apartado 4 se actualizan las reglas de sintonización a los controladores PID Ideal y Serie actuales y a la utilización de los modelos de primer y segundo orden más tiempo muerto, identificados a partir de la curva de reacción del proceso; y en el apartado 5 se presenta un ejemplo ilustrativo de la utilización de las ecuaciones actualizadas.

\section{MÉTODO DE SINTONIZACIÓN DE ZIEGLER Y NICHOLS}

Ziegler y Nichols realizaron pruebas de simulación y experimentales con equipo de su laboratorio, para determinar los parámetros de controladores P, PI y PID.

Empezando con el controlador puramente proporcional, encontraron que la selección de la sensitividad (ganancia) adecuada del controlador, requería de un compromiso entre la razón de amplitud de la respuesta (decaimiento del error en un periodo) y el error permanente. Consideraron entonces que un decaimiento del $25 \%$, proporcionaba un compromiso adecuado y que este se lograba, con una sensitividad aproximadamente igual a la mitad de la sensitividad máxima.

Para los modos integral y derivativo, encontraron que su ajuste podía relacionarse con el periodo de oscilación crítico, para lograr siempre el decaimiento recomendado del $25 \%$. A partir de ahí, dedujeron las ecuaciones de sintonización de los controladores.

\subsection{Método de lazo cerrado}

Las ecuaciones de sintonización de Ziegler y Nichols, para el controlador Fulscope 100, empleando la información crítica o última $\left(\mathrm{K}_{\mathrm{cu}}\right.$, $\mathrm{T}_{\mathrm{u}}$ ) son:

- Controlador P

$K_{c}=0,50 K_{c u}$
- Controlador PI

$K_{c}=0,45 K_{c u}$

$T_{i}=T_{u} / 1,2$

- Controlador PID

$$
\begin{gathered}
K_{c}=0,6 K_{c u} \quad a \quad 1,0 K_{c u} \\
T_{i}=T_{u} / 2 \\
T_{d}=T_{u} / 8
\end{gathered}
$$

\subsection{Método de lazo abierto}

En adición a las pruebas que les condujeron a la determinación de las ecuaciones del método de lazo cerrado anterior, Ziegler y Nichols obtuvieron la curva de respuesta de los sistemas probados, a un cambio escalón en la entrada, denominada curva de reacción. Trazando una recta tangente al punto de inflexión de dicha respuesta, como se observa en la Figura 1, determinaron el retraso $\mathrm{L}$ del sistema y su razón de reacción $\mathrm{R}$ (pendiente de la recta). Las pruebas experimentales les permitieron determinar que el periodo de oscilación del sistema con un controlador P, era aproximadamente cuatro veces el valor del retraso $\left(\mathrm{T}_{\mathrm{u}} \approx 4 \mathrm{~L}\right)$ y que la sensitividad máxima, era inversamente proporcional al producto $R L$ $\left(\mathrm{K}_{\mathrm{cu}} \approx 2 / \mathrm{RL}\right)$. Empleando estas relaciones en las ecuaciones de la (1) a la (3), encontraron los parámetros del controlador Fulscope 100, en función de las características de la respuesta al escalón, como:

\section{- Controlador $P$}

$K_{c}=1 / R L$

- Controlador PI

$K_{c}=0,9 / R L$

$T_{i}=L / 0,3$ 
- Controlador PID

$$
\begin{gathered}
K_{c}=1,2 / R L \quad a \quad 2,0 / \mathrm{RL} \\
T_{i}=2,0 L \\
T_{d}=0,5 L
\end{gathered}
$$

\section{CARACTERÍSTICAS DEL MÉTODO DE ZIEGLER Y NICHOLS}

A continuación se detallan las características principales de este método de sintonización.

\subsection{Tipo de funcionamiento del lazo de control}

Ziegler y Nichols realizaron todas las pruebas, introduciendo un cambio escalón en la salida del controlador, entrada a la planta, manteniendo en todo momento el valor deseado constante. Es importante tener en cuenta entonces, que estas reglas de sintonización solo son aplicables para la sintonización de controladores que operarán como reguladores, y no como servomecanismos.

Es muy frecuente encontrar en la literatura técnica, propuestas de nuevas reglas de sintonización en las cuales se comparan estas, con las respuestas obtenidas con el método de Ziegler y Nichols ante un cambio escalón en el valor deseado, para demostrar su supuesta superioridad. Estas comparaciones no tienen validez, ya que Ziegler y Nichols no se preocuparon nunca, por la respuesta del sistema de control a un cambio en el valor deseado.

\subsection{Criterio de desempeño}

El criterio de desempeño seleccionado por Ziegler y Nichols fue el decaimiento del $25 \%$ para un cambio escalón en la carga, esto es, que la amplitud del error máximo decayera a su cuarta parte en cada periodo de oscilación.

\subsection{Información para el método de lazo cerrado}

El método de lazo cerrado requiere conocer los parámetros críticos, ganancia $K_{c u}$ y periodo de oscilación $T_{w}$, obtenidos, según el procedimiento original, llevando el sistema al límite de la estabilidad, utilizando un controlador puramente proporcional.

\subsection{Información para el método de lazo abierto}

Como se indicó anteriormente, para la utilización de las relaciones a partir de la información obtenida de una prueba de lazo abierto, es necesario trazar una recta tangente al punto de inflexión de la misma, para determinar el retraso (tiempo muerto aparente) y la pendiente de la recta.

En ningún momento Ziegler y Nichols identificaron un modelo de primer orden más tiempo muerto, a partir de la curva de reacción, ni consideraron que el proceso fuera autorregulado, esto es, que alcanzara un nuevo punto de equilibrio. Un modelo de la planta que se adecua más a la información obtenida a partir de la recta tangente, es el de un proceso integrante dado por la ecuación:

$G_{p}(\mathrm{~s})=\frac{R e^{-L s}}{s}$

Es muy frecuente encontrar que las ecuaciones de sintonización de Ziegler y Nichols de lazo abierto, se emplean con los parámetros de un modelo de primer orden, más tiempo muerto de la forma

$G_{p 0}(s)=\frac{K_{p} e^{-L s}}{T s+1}$

en donde $K_{p}$ es la ganancia estática, $T$ la constante de tiempo y $L$ el tiempo muerto aparente.

Para que este modelo sea válido, para emplearlo en la sintonización de los parámetros del controlador utilizando las reglas de Ziegler y Nichols, su tiempo muerto L y constante de tiempo $\mathrm{T}$, se deben determinar empleando la 
recta tangente como se muestra en la Figura 1. El valor de la constante de tiempo obtenida de esta forma, es mayor que el calculado si se identifica el modelo de manera que reproduzca con mayor fidelidad la respuesta dinámica de la planta y por lo tanto, dará valores diferentes para los parámetros del controlador.

Las ecuaciones de sintonización de Ziegler y Nichols para el controlador Fulscope 100, utilizando los parámetros de un modelo de primer orden más tiempo muerto (8), identificado por medio de la tangente son, entonces:

- $\quad$ Controlador P

$K_{c}=T / K_{p} L$

- Controlador PI

$K_{c}=0,9 \mathrm{~T} / K_{p} L$

$T_{i}=L / 0,3$

- Controlador PID

$$
\begin{gathered}
K_{c}=1,2 T / K_{p} L \quad a \quad 2,0 T / K_{p} L \\
T_{i}=2,0 L \\
T_{d}=0,5 L
\end{gathered}
$$

\subsection{Tipo de controlador PID}

Si bien el controlador PID empleado por Ziegler y Nichols fue un Taylor Fulscope 100, existen opiniones encontradas sobre el tipo de controlador PID actual, al que se pueden aplicar directamente las ecuaciones de sintonización determinadas por ellos.

Por un lado están los que afirman que estas son para controladores PID tipo Serie, o interactuantes, ya que los controladores neumáticos antiguos tenían supuestamente esta forma (Corripio, 2001), (Harrold, 1999), (VanDoren, 1998). Otros, por el contrario, indican que son aplicables a controladores PID tipo Ideal, o no interactuantes (Goodwing et al., 2001), (Hang et al., 1993), (O’Dwyer., 2003) y (Tan et al., 1999).

Para aclarar este punto, fue necesario determinar cuál era la función de transferencia aproximada del controlador Taylor Fulscope 100 empleado por Ziegler y Nichols.

Según indican (Farrigton, 1951) y (Harriot, 1964) los controladores neumáticos antiguos, tenían una ecuación de la forma:(ver ecuación 12) en donde $\mathrm{k}_{\mathrm{c}}, \tau_{\mathrm{i}} \mathrm{y} \tau_{\mathrm{d}}$ correspondían a los ajustes de las perillas del controlador. Esto es reafirmado por Coughanour y Koppel (1965) quienes indican que la ecuación del controlador Taylor Fulscope 100 era de la forma (ver ecuación 12).

$$
\begin{gathered}
u(s)=k_{c}\left(\frac{\tau_{i}+\tau_{d}}{\tau_{i}-\tau_{d}}\right)\left[1+\frac{1}{\left(\tau_{i}+\tau_{d}\right) s}+\frac{\tau_{i} \tau_{d}}{\tau_{i}+\tau_{d}} s\right] e(s) \\
u(s)=k_{c}\left(\frac{1+\tau_{d} / \tau_{i}}{1-\tau_{d} / \tau_{i}}\right)\left[1+\frac{1}{\left(1+\tau_{d} / \tau_{i}\right) \tau_{i} s}+\frac{\tau_{d}}{1+\tau_{d} / \tau_{i}} s\right] e(s)
\end{gathered}
$$


Esta ecuación se puede reordenar como (ver ecuación 13)

Entonces, los parámetros efectivos del controlador Fulscope 100 eran:

$K_{c}=k_{c}\left(\frac{1+\tau_{d} / \tau_{i}}{1-\tau_{d} / \tau_{i}}\right)$

$T_{i}=\left(1+\tau_{d} / \tau_{i}\right) \tau_{i}$

$T_{d}=\frac{\tau_{d}}{\left(1+\tau_{d} / \tau_{i}\right)}$

Las expresiones anteriores para el cálculo del tiempo integral $T_{i}$ y el tiempo derivativo $T_{d}$ efectivos del controlador, coinciden con las ecuaciones utilizadas para la conversión de los parámetros de un controlador PID Serie a uno Ideal. Sin embargo, la ecuación para la ganancia proporcional efectiva $K_{c}$, incluye además, el término $\left(1-\tau_{d} / \tau_{i}\right)$ en el denominador. Este factor aumenta la ganancia proporcional equivalente del controlador, aspecto que, no se ha encontrado, haya sido tomado en consideración en la utilización de las ecuaciones de Ziegler y Nichols con controladores diferentes del Fulscope 100.

El factor de interacción $\left(1+\tau_{d} / \tau_{i}\right)$ para el controlador Fulscope 100, con la sintonización de Ziegler y Nichols es entonces, 1,25.

\section{ACTUALIZACIÓN DE LAS REGLAS DE SINTONIZACIÓN}

Como el desarrollo tecnológico ha hecho que los controladores PID actuales, tengan una función de transferencia diferente de la del empleado por Ziegler y Nichols en sus pruebas, y que en la actualidad se determinen modelos de primer orden más tiempo muerto para representar el proceso, por procedimientos de identificación diferentes del de la tangente, es necesario actualizar las relaciones determinadas por Ziegler y Nichols, para poder obtener características de respuesta del lazo de control, de conformidad con el criterio de desempeño seleccionado por ellos.

\subsection{Controladores $P I D$}

Los controladores $P$ y $P I$ presentan la misma función de transferencia, independientemente de su realización física, por lo que las ecuaciones de sintonización para estos, no se ven afectadas por la evolución tecnológica.

El caso de los controladores PID es completamente diferente. Desde la construcción del primer controlador PID, el Taylor Fulscope 100, ha habido diferencias entre las expresiones utilizadas en los libros de texto para explicar su funcionamiento y su realización por parte de los fabricantes.

Se puede decirque existen tres expresiones básicas, para la representación de los controladores PID:

- PIDIdeal

$u(s)=K_{c}\left[1+\frac{1}{T_{i} s}+T_{d} s\right] e(s)$

- $\quad$ PIDSerie

$u(s)=K_{c}^{\prime}\left[1+\frac{1}{T_{i}^{\prime} s}\right]\left[1+T_{d}^{\prime} s\right] e(s)$

- PIDParalelo

$u(s)=\left[K_{p}+\frac{K_{i}}{s}+K_{d} s\right] e(s)$

existiendo además variaciones de estas que contemplan la realización de un derivador real, la aplicación del modo derivativo solo a la señal realimentada y otras. De estas, la utilizada con mayor frecuencia por los fabricantes, es el controlador denominado PIDIndustrial, el cual está dado por la ecuación: 
$u(s)=K_{c}^{\prime}\left\{\left[1+\frac{1}{T_{i} s}\right] r(s)-\left[1+\frac{1}{T_{i} s}\right]\left[1+T_{d}^{\prime} s\right] y(s)\right\}$

Utilizando las ecuaciones (3), (6), (11) y (14), se puede determinar que los parámetros de un controlador PID actual, sintonizado con el método de Ziegler y Nichols utilizando la información última, deben ser, entonces:

- PIDIdeal

$$
\begin{gathered}
K_{c}=K_{c u} \quad a \quad 1,67 K_{c u} \\
T_{i}=T_{u} / 1,6 \\
T_{d}=T_{u} / 10
\end{gathered}
$$

- PIDSerie o Industrial

$$
\begin{gathered}
K_{c}^{\prime}=0,80 K_{c u} \quad a \quad 1,33 K_{c u} \\
T_{i}^{\prime}=T_{u} / 2 \\
T_{d}^{\prime}=T_{u} / 8
\end{gathered}
$$

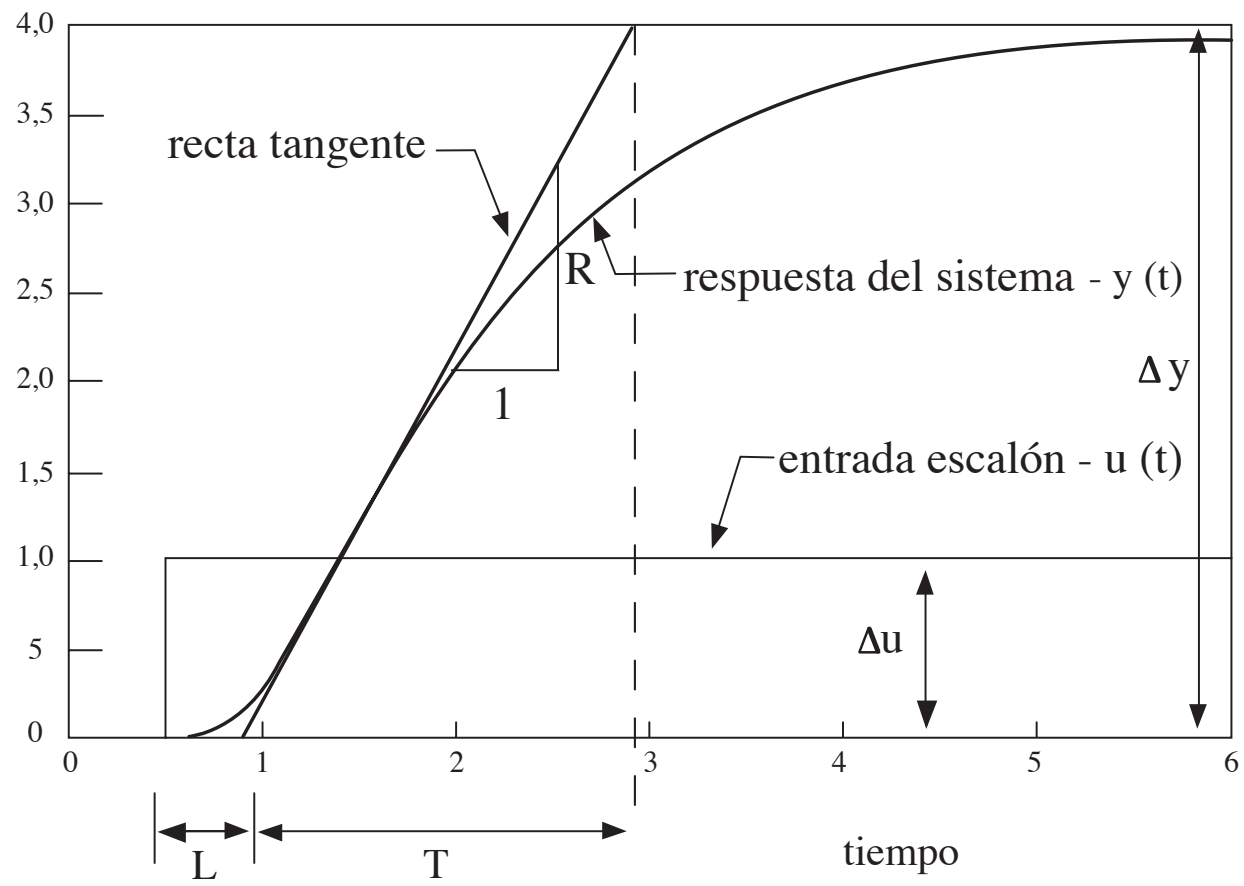

Figura 1. Curva de reacción y recta tangente.

$$
\text { Fuente: (El autor) }
$$

En el caso de la información obtenida a partir de la curva de reacción del proceso controlado, por el método de la tangente, los parámetros para un controlador PID actual serían:

- PIDIdeal

$$
\begin{array}{ccc}
K_{c}=2,0 / R L & a & 3,33 / R L \\
K_{c}=2,0 T / K_{p} L & a & 3,33 T / K_{p} L \\
T_{i}=2,5 L & \\
T_{d}=0,4 L &
\end{array}
$$

- PIDSerie o Industrial

$$
\begin{aligned}
& K_{c}^{\prime}=1,6 / R L \quad a \quad 2,67 / R L \\
& K_{c}^{\prime}=1,6 T / K_{p} L \quad a \quad 2,67 T / K_{p} L \\
& T_{i}^{\prime}=2,0 L \\
& T_{d}^{\prime}=0,5 L
\end{aligned}
$$




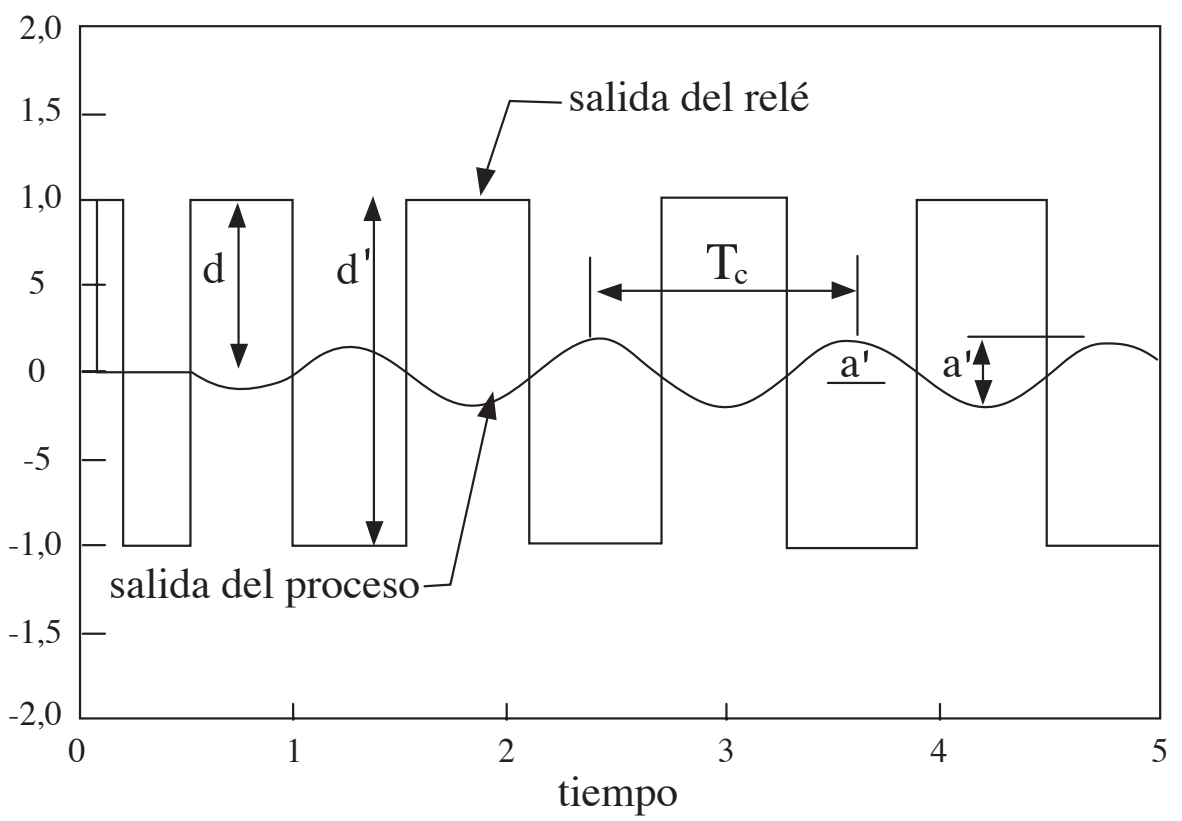

Figura 2. Respuesta del sistema de control con relé.

Fuente: (El autor)

\subsection{Información para el método de lazo cerrado}

En el procedimiento original era necesario llevar el sistema de control al límite de la estabilidad, utilizando un controlador proporcional, para determinar los parámetros $K_{c u}$ y $T_{u}$. Como esto normalmente no es deseable, e incluso muchas veces no es posible, esta información se puede obtener con bastante precisión empleando un relé para controlar el sistema, como lo propusieron Aström y Hägglund (Aström \& Hägglund, 1984) presentado en la Figura 2. En este caso, los parámetros últimos se pueden determinar como

$$
\begin{gathered}
K_{c u}=\frac{4 d}{\pi a}=\frac{4 d^{\prime}}{\pi a}, \\
T_{u}=T_{c}
\end{gathered}
$$

\subsection{Identificación del modelo a lazo abierto}

Como se ha indicado anteriormente, Ziegler y Nichols nunca identificaron un modelo de primer orden más tiempo muerto, a partir de la curva de reacción. Ellos basaron sus relaciones de lazo abierto, en la observación de que el periodo de oscilación del sistema con un controlador $P$, era aproximadamente cuatro veces el tiempo muerto aparente de la planta.

Por esta razón, no es correcto emplear los parámetros de un modelo de primer orden más tiempo muerto, identificado a partir de la curva de reacción por un método que no sea el de la tangente, directamente en las ecuaciones de Ziegler y Nichols. Es muy probable también, que ya solo se cuente con el modelo obtenido utilizando algún programa de identificación y no con la curva de reacción original, por lo que es necesario adecuar las ecuaciones de Ziegler y Nichols para poder ser empleadas con estos modelos.

Para verificar el efecto que la identificación de las características dinámicas del proceso pudiera tener sobre el comportamiento del lazo de control, se realizó una prueba comparativa empleando la planta (A1) descrita en el Anexo A y sintonizando un controlador PIDIdeal con la información última (A2), la información 
obtenida de la curva de reacción por el método de la tangente (A3) y un modelo de primer orden más tiempo muerto, identificado con un método de dos puntos (A4). Los parámetros del controlador se muestran en el Cuadro 1 y en la Figura 3, las respuestas de estos tres sistemas.

Cuadro 1. Parámetros de Ziegler y Nichols para el controlador PIDIdeal

\begin{tabular}{|c|c|c|c|}
\hline & $\begin{array}{l}\text { Información } \\
\text { última }\end{array}$ & $\begin{array}{l}\text { Método de la } \\
\text { tangente }\end{array}$ & $\begin{array}{l}\text { Modelo de } \\
\text { primer orden }\end{array}$ \\
\hline & Ecuación (19) & Ecuación (21) & Ecuación (21) \\
\hline & 6,75 & 8,76 & 3,61 \\
\hline . & 1,39 & 1,25 & 1,73 \\
\hline & 0,22 & 0,20 & 0,28 \\
\hline
\end{tabular}

Fuente: (El autor)

Tomando como correctos los parámetros del controlador calculados con la información última, se nota que los parámetros obtenidos con el modelo identificado por el método de la tangente difieren de estos (ganancia $30 \%$ mayor, tiempo integral $10 \%$ menor, tiempo derivativo $10 \%$ menor). Al tener el controlador más acción integral y menos acción derivativa, la respuesta del sistema es más oscilatoria, con un tiempo de asentamiento mayor.

La razón por la cual los parámetros calculados utilizando la información obtenida con la tangente, difieren de los determinados con la información crítica, es consecuencia de que, para la planta de prueba utilizada, no se cumplen los supuestos hechos por Ziegler y Nichols e indicados en el apartado 2.2, para el desarrollo de su método de lazo abierto; en este caso $T_{u}>4 t_{m}$ y $K_{c u}<2 / R L$.

Por otra parte, los parámetros obtenidos utilizando el modelo de primer orden más tiempo muerto, identificado con un método de dos puntos, difieren aún más (ganancia $47 \%$ menor, tiempo integral $24 \%$ mayor, tiempo derivativo $27 \%$ mayor). Al tener el controlador menos ganancia y menos acción integral, la respuesta del sistema de control es muy poco amortiguada, mostrando una tendencia no oscilatoria y lenta.
Es necesario por lo tanto modificar las ecuaciones de lazo abierto originales de Ziegler y Nichols, para que se puedan utilizar con un modelo obtenido a partir de la curva de reacción, por procedimientos distintos del método de la tangente.

Para una planta de primer orden más tiempo muerto con un controlador proporcional, se tiene que en el límite de la estabilidad

$$
\begin{aligned}
& \frac{K_{c} k_{p}}{\sqrt{\left(\tau \omega_{u}\right)^{2}+1}}=1 \\
& -t_{m} \omega_{u}-\tan ^{-1}\left(\tau \omega_{u}\right)=-\pi
\end{aligned}
$$

Sustituyendo $\omega_{\mathrm{u}}=2 \pi / \mathrm{T}_{\mathrm{u}}$ en (24) y (25) se obtiene que

$$
\begin{aligned}
& K_{c u}=\frac{\sqrt{\left(2 \pi \tau / T_{u}\right)^{2}+1}}{k_{p}} \\
& -\frac{2 \pi t_{m}}{T_{u}}-\tan ^{-1}\left(\frac{2 \pi \tau}{T_{u}}\right)=-\pi
\end{aligned}
$$

La ecuación (26) permite determinar en forma explícita la ganancia límite del controlador $K_{c u}$, a partir de los parámetros del modelo, una vez conocido el periodo de oscilación. Sin embargo, el periodo de oscilación $T_{u}$ requiere de la solución numérica de (27).

En vez de emplear las expresiones (26) y (27) anteriores, utilizando Matlab® se investigó el comportamiento del sistema de control en el límite de la estabilidad para el ámbito de $0,1 \leq t_{m} / \tau \leq 1,0$, y se determinaron ecuaciones para la estimación de los parámetros últimos, a partir de un modelo de primer orden más tiempo muerto de la forma

$G_{p 1}(s)=\frac{k_{p} e^{-t_{m} s}}{\tau_{s}+1}$ 
Las expresiones obtenidas son:

$$
\begin{aligned}
k_{p} K_{c u} & =0,68+1,56\left(\tau / t_{m}\right) \\
\frac{T_{u}}{t_{m}} & =\frac{1,88+0,72\left(t_{m} / \tau\right)}{0,47+0,37\left(t_{m} / \tau\right)}
\end{aligned}
$$

La ecuación (29) indica que $T_{u} 4 t_{m}$ si $t_{m}<<\tau$, pero que $T_{u} \mathrm{~g} 3.1 t_{m}$ si $t_{m} \tau$. Por lo tanto, la relación $T_{u} \approx 4 L$ empleada por Ziegler y Nichols, no se puede considerar como constante.

Un trabajo similar se realizó también a partir de un modelo de segundo orden (polo doble) más tiempo muerto, dado por

$G_{p 2}(s)=\frac{k_{p} e^{-t_{m}^{\prime} s}}{\left(\tau^{\prime} s+1\right)^{2}}$

determinándose las siguientes expresiones:

$$
\begin{gathered}
k_{p} K_{c u}=0,70+2,0\left(\tau^{\prime} / t_{m}^{\prime}\right) \\
\frac{T_{u}}{t^{\prime}{ }_{m}}=\frac{1,75+2,20\left(t_{m}^{\prime} / \tau^{\prime}\right)}{0,066+0,75\left(t^{\prime}{ }_{m} / \tau^{\prime}\right)}
\end{gathered}
$$

Las ecuaciones (29) o (31) permiten estimar los parámetros últimos del sistema de control, con un controlador proporcional y el modelo identificado a partir de la curva de reacción, y utilizarlos en las ecuaciones de cálculos de los parámetros del controlador, (19) o (20) dependiendo del controlador que se deba sintonizar.

En el caso de utilizar un modelo de primer orden más tiempo muerto (28), los parámetros de un controlador PID actual deben ser:

- PIDIdeal

$$
\begin{aligned}
K_{c}=\frac{K}{k_{p}} & {\left[0,68+\frac{1,56}{\tau_{o}}\right], 1,0 \leq K \leq 1,67 } \\
T_{i} & =\left[\frac{2,50+0,957 \tau_{o}}{1+0,787 \tau_{o}}\right] t_{m} \\
T_{d} & =\left[\frac{0,40+0,153 \tau_{o}}{1+0,787 \tau_{o}}\right] t_{m}
\end{aligned}
$$

- PIDSerie o Industrial:

$$
\begin{gathered}
K_{c}^{\prime}=\frac{K}{k_{p}}\left[0,68+\frac{1,56}{\tau_{o}}\right], 0,8 \leq K \leq 1,33 \\
T_{i}^{\prime}=\left[\frac{2,0+0,766 \tau_{o}}{1+0,787 \tau_{o}}\right] t_{m} \\
T_{d}^{\prime}=\left[\frac{0,50+0,191 \tau_{o}}{1+0,787 \tau_{o}}\right] t_{m}
\end{gathered}
$$

en donde $\tau_{o}=t_{m} / \tau$.

Si se cuenta con un modelo de segundo orden (polo doble) más tiempo muerto (30), los parámetros de un controlador PID actual deben ser:

- $\quad$ PIDIdeal

$K_{c}=\frac{K}{k_{p}}\left[0,70+\frac{2,0}{\tau_{o}^{\prime}}\right], 1,0 \leq K \leq 1,67$

$$
\begin{aligned}
& T_{i}=\left[\frac{16,57+20,83 \tau_{o}^{\prime}}{1+11,36 \tau_{o}^{\prime}}\right] t_{m}^{\prime} \\
& T_{d}=\left[\frac{2,65+3,33 \tau_{o}^{\prime}}{1+11,36 \tau_{o}^{\prime}}\right] t_{m}^{\prime}
\end{aligned}
$$

- PIDSerie o Industrial:

$$
\begin{gathered}
K_{c}^{\prime}=\frac{K}{k_{p}}\left[0,70+\frac{2,0}{\tau_{o}^{\prime}}\right], 0,8 \leq K \leq 1,33 \\
T_{i}^{\prime}=\left[\frac{13,26+16,67 \tau_{o}^{\prime}}{1+11,36 \tau_{o}^{\prime}}\right] t_{m}^{\prime} \\
T_{d}^{\prime}=\left[\frac{3,31+4,17 \tau_{o}^{\prime}}{1+11,36 \tau_{o}^{\prime}}\right] t_{m}^{\prime}
\end{gathered}
$$

en donde $\tau_{o}^{\prime}=\mathrm{t}_{m}^{\prime} / \tau^{\prime}$.

\section{USO DE LAS ECUACIONES ACTUALIZADAS}

Como ejemplo de la aplicación de las ecuaciones actualizadas derivadas para el método de sintonización de Ziegler y Nichols, (32) a (35), se empleó nuevamente la planta descrita en el Apéndice. 
Los parámetros últimos obtenidos a partir del modelo de primer orden más tiempo muerto (A4) y los predichos con las ecuaciones (29) a partir de este modelo, se muestran en el Cuadro 2.

Cuadro 2. Parámetros últimos a partir del modelo de primer orden más tiempo muerto

$\mathrm{K}_{\mathrm{cu} 1}$

Identificados
3,50
2,33

Predichos

3,50

$\mathrm{T}_{\mathrm{u} 1}$

Fuente: (El autor)

En el Cuadro 3 se muestran los parámetros últimos determinados, utilizando el modelo de segundo orden (A5) y los predichos para este modelo por las ecuaciones (31).

Cuadro 3. Parámetros últimos a partir del modelo de segundo orden (polo doble) más tiempo muerto

$\begin{array}{lcc} & \text { Identificados } & \text { Predichos } \\ \mathrm{K}_{\mathrm{cu} 2} & 6,16 & 6,17 \\ \mathrm{~T}_{\mathrm{u} 2} & 2,19 & 2,17 \\ & \text { Fuente: (El autor) } & \end{array}$

Como se puede apreciar en los cuadros anteriores, las ecuaciones derivadas para la determinación de los parámetros últimos son adecuadas, tanto para el modelo de primer orden como el de polo doble. Sin embargo, estos no necesariamente son buenos, comparados con los valores obtenidos empleando la planta de prueba (A2).

Los modelos obtenidos a partir de la curva de reacción, normalmente no modelan adecuadamente el comportamiento del sistema real en el punto de oscilación mantenida. Si bien la estimación del periodo de oscilación hecha con ambos modelos se puede considerar buena, en este caso, la estimación de la ganancia crítica a partir del modelo de primer orden, es solo el $52 \%$ del valor real, mientras que la estimada a partir del modelo de segundo orden, alcanza el $91 \%$.

Los parámetros para un controlador PIDIdeal calculados con la información anterior se muestran en el Cuadro 4.
Cuadro 4. Parámetros de Ziegler y Nichols para el controlador PIDIdeal

$\begin{array}{ccc}\begin{array}{c}\text { Información } \\ \text { última } \\ \text { Ecuación (19) }\end{array} & \begin{array}{c}\text { Modelo de } \\ \text { primer orden } \\ \text { Ecuación (32) }\end{array} & \begin{array}{c}\text { Modelo de } \\ \text { segundo orden } \\ \text { Ecuación(34) }\end{array} \\ 6,75 & 3,50 & 6,17 \\ 1,39 & 1,46 & 1,36 \\ 0,22 & 0,23 & 0,22 \\ & \text { Fuente: (El autor) }\end{array}$

Como se puede apreciar en este cuadro, la diferencia entre los parámetros es significativa solo para el valor de la ganancia proporcional obtenida utilizando el modelo de primer orden más tiempo muerto, debido a que este modelo no permite determinar adecuadamente la ganancia crítica del sistema.

En la Figura 4 se muestran las respuestas del sistema de control, con el controlador PIDIdeal sintonizado con los tres conjuntos de parámetros anteriores.

Comparando las diferentes respuestas mostradas en las Figuras 3 y 4, con la obtenida con el controlador sintonizado con los parámetros últimos, curva 1 , se nota que la respuesta del sistema sintonizado con base en la información obtenida por el método de la tangente, curva 2, tiene una amplitud ligeramente mayor, pero es más oscilatoria, como se destacó anteriormente. La respuesta obtenida con los parámetros del controlador calculados con el modelo de primer orden utilizando las ecuaciones actualizadas, curva 4, es mucho mejor que la obtenida con estos parámetros empleando las ecuaciones originales, curva 3. Además, la respuesta del sistema sintonizado utilizando las ecuaciones actualizadas y un modelo de segundo orden, curva 5, no solo es mejor que la respuesta obtenida utilizando el modelo de primer orden, curva 4, si no que se aproxima con bastante exactitud a la respuesta obtenida con el controlador sintonizado con la información última, curva 1. Se puede verificar que, si la ganancia del controlador sintonizado con el modelo de segundo orden y las ecuaciones actualizadas se incrementa en solo un $10 \%$, la 
diferencia de su respuesta con la obtenida con los parámetros últimos es imperceptible.

Si se cuenta con un modelo de primer orden, se puede sintonizar el controlador con las ecuaciones actualizadas, e incrementar su ganancia; si fuera necesario, se podría reducir el error máximo para lograr la respuesta deseada.

Considerando lo anterior, para utilizar las reglas de sintonización de Ziegler y Nichols, se recomienda emplear la información última del proceso obtenida mediante la prueba del relé, y de ser necesario, con base en un modelo identificado a partir de la curva de reacción, emplear las ecuaciones actualizadas, preferiblemente con un modelo de segundo orden (polo doble).

\section{CONCLUSIONES}

Las ecuaciones de sintonización de controladores $P I D$ de Ziegler y Nichols fueron obtenidas hace más de sesenta años. El avance tecnológico en la fabricación de los controladores, o su programación, hace que las ecuaciones de los mismos difieran de las del controlador utilizado originalmente en las pruebas para la obtención de estas reglas de sintonización. Además, las técnicas de identificación empleadas actualmente para la obtención de un modelo de orden reducido para el sistema, difieren sustancialmente de las empleadas por Ziegler y Nichols. Esto hace necesario una actualización de estas reglas de sintonización, para obtener sistemas de control con las características dinámicas recomendadas por estos autores.

El desarrollo de las ecuaciones de Ziegler y Nichols actualizadas permitió determinar, además, un procedimiento novedoso para la aplicación de este método a la sintonización de los controladores PID, a partir de un modelo de segundo orden (polo doble). Estas ecuaciones demostraron dar resultados comparables con los obtenidos a partir de la información última.

Para la utilización del criterio de sintonización de Ziegler y Nichols a los controladores PID modernos y con modelos identificados por técnicas diferentes de la tangente, se requiere por

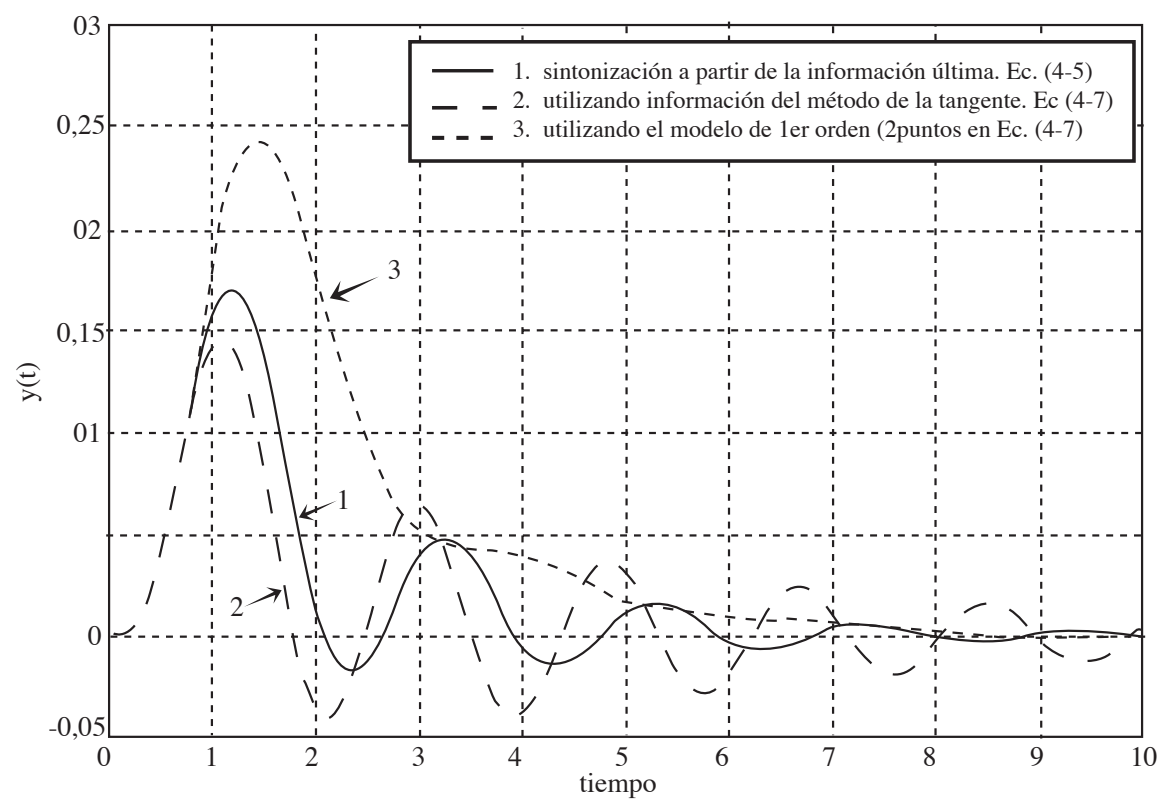

Figura 3. Respuesta del sistema de control, PIDIdeal.

Fuente: (El autor) 


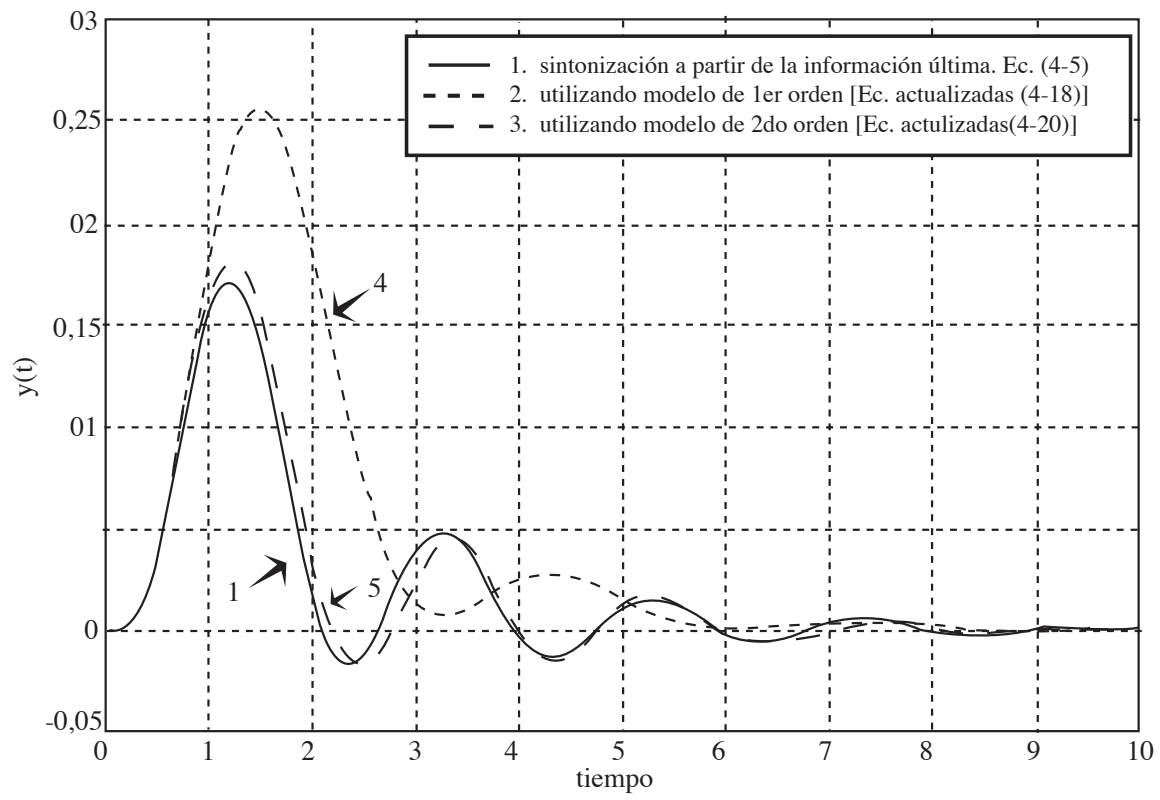

Figura 4. Respuesta del sistema de control, PIDIdeal.

Fuente: (El autor)

lo tanto emplear las ecuaciones de sintonización actualizadas presentadas, (19), (20), o (32) a (35), según sea el controlador y la información disponible del sistema.

Debe tenerse en consideración también, que como con cualquier regla de sintonización, los parámetros calculados deben considerarse solo una primera aproximación de los valores requeridos, los que usualmente requieren de un afinamiento posterior, para lograr el comportamiento deseado del sistema de control con el proceso real.

\section{SIMBOLOGÍA}

$G_{c}(s)$ función de transferencia del controlador

$G_{p}(s)$ función de transferencia de la planta

$K_{c} \quad$ ganancia del controlador

$K_{c u} \quad$ ganancia crítica

$k_{p} \quad$ ganancia de la planta

$L \quad$ retardo

$R \quad$ pendiente de la recta tangente

$s \quad$ variable compleja
T constante de tiempo

$T_{d} \quad$ tiempo derivativo

$T_{i} \quad$ tiempo integral

$t_{m} \quad$ tiempo muerto aparente

$T_{u} \quad$ periodo de oscilación crítico

$\tau \quad$ constante de tiempo

\section{REFERENCIAS BIBLIOGRÁFICAS}

Aström, K. J. \& Hägglund, T. (1984). Automatic tuning of simple regulators with specifications on phase and amplitude margins. Automatica, 5(20), 645-651.

Babb, M. (1990). Pneumatic Instruments Gave Birth to Automatic Control. Control Engineering, October.

Bickley, G. (1990). Modern Control Started with Ziegler y Nichols Tuning. Control Engineering, October.

Corripio, A. B. (2001). Tuning of Industrial Control Systems, Research Triangle Park, NC, 
E.U.A.:ISA - The Instrumentation, Systems and Automation Society.

Coughanour, D. R. \& Koppel, L. B. (1965). Process Systems Analysis and Control. McGrawHill, Inc.

Farrigton, G. H. (1951). Fundamentals of Automatic Control. John Wiley \& Sons.

Goodwing, G. C., Graebe, S. F. \& Salgado, M. E. (2001). Control Systems Design. Upper Saddle River, NJ, E.U.A.: PrenticeHall, Inc.

Hang, C. C., Lee, T. H. \& Ho, W. K. (1993). Adaptive Control. Research Triangle Park, NC, E.U.A.: Instrument Society of America.

Harriot, P. (1964). Process Control. McGrawHill Book Co.

Harrold, D. (1999). Process Controller Tuning Guidelines. Control Engineering (web exclusive), August.

O'Dwyer, A. (2003). Handbook of PI and PID Controller Tuning Rules, London.: Imperial College Press.
Tan, K. K., QG. Wang, C. C. Hang \& T. J. Hägglund. (1999). Advances in PID Control, London: SpringerVerlag.

VanDoren, V. J. (1998). Ziegler y Nichols Methods Facilitate Loop Tuning. Control Engineering, August.

Ziegler, J. G. \& Nichols, N. B. (1942). Optimum Settings for Automatic Controllers. ASME Transactions, 64.

\section{SOBRE EL AUTOR}

\section{Víctor M. Alfaro Ruiz}

Ingeniero. Eléctrico. Profesor Asociado, Departamento de Automática, Escuela de Ingeniería Eléctrica, Universidad de Costa Rica. Teléfono: 207-4472

Facsímil: 207-4139

Apartado postal: 210, 2060, San José, Costa Rica. Correo electrónico: valfaro@eie.ucr.ac.cr

Este trabajo fue realizado como parte del Proyecto de Investigación $N^{o} 32243007$, inscrito en la Vicerrectoría de Investigación de la Universidad de Costa Rica. 


\section{ANEXO A}

Para las pruebas comparativas realizadas con el método de Ziegler y Nichols se utilizó como planta:

$$
G_{p}(s)=\frac{1}{(s+1)(0,5 s+1)(0,25 s+1)(0,125 s+1)}
$$

Los parámetros últimos para el sistema de control proporcional de esta planta son:

$$
K_{c u}=6,75, T_{u}=2,22
$$

Utilizando el método de la tangente, a partir de la curva de reacción se identificó el siguiente modelo para el proceso

$$
G_{m 0}(s)=\frac{e^{-0,50 s}}{2,19 s+1}
$$

y utilizando el método de dos puntos "1/4 - 3/4", los siguientes dos modelos:

- Primer orden más tiempo muerto

$G_{m 1}(s)=\frac{e^{-0,691 s}}{1,247 s+1}$

- Segundo orden (polo doble) más tiempo muerto

$$
G_{m 2}(s)=\frac{e^{-0,289 s}}{(0,791 s+1)^{2}}
$$

